\title{
O USO DA INTELIGÊNCIA ARTIFICIAL PELO SISTEMA JURÍDICO BRASILEIRO, CLASSIFICAÇÃO DA INTELIGÊNCIA ARTIFICIAL E ANÁLISE DE SEU USO
}

\author{
THE USE OF ARTIFICIAL INTELLIGENCE BY THE JUDICIARY BRANCH, \\ CLASSIFICATION OF THE ARTIFICIAL INTELLIGENCE AND ANALYSIS OF IT'S \\ USE
}

Matheus Viezzer ${ }^{1}$

RESUMO: Os avanços tecnológicos tem influenciado cada vez mais a vida em sociedade, alterando as formas de relacionamento e contato entre os indivíduos, alcançando a esfera das profissões, substituindo e realocando o trabalho antes exercido por força ou cognição humana, esta força crescente é denominada Inteligência Artificial, e os avanços tecnológicos constantes desta geram a urgência de seu estudo para sua melhor compreensão. Assim, este trabalho se destina a apresentar o funcionamento e classificação de sistemas inteligentes artificialmente, realizando explicitação de conceitos presentes no instituto da Inteligência Artificial. Tal análise fora realizada buscando compreender os programas artificialmente inteligentes empregados pelo Poder Judiciário, sua classificação dentro da Inteligência Artificial, e se estes estão sendo utilizados de forma a compreender todas suas habilidades e funções. O presente trabalho se utilizou da análise do material literário específico, assim como de estudos em sítios eletrônicos respectivos de cada projeto ou conceito analisado, realizando pesquisa bibliográfica e descritiva, analisando as matérias com maior aprovação pela comunidade científica sobre o tema, e apresentando discussão sobre as aplicações destas concepções sobre o modelo utilizado no Poder Judiciário brasileiro, a fim de verificar se estes utilizam Inteligência Artificial ou são somente utilizadas para automação de funções.

Palavras-chave: Avanços tecnológicos. Inteligência Artificial. Compreensão. Classificação. Poder Judiciário.

ABSTRACT: The technological advances has been influenced our life in society more and more, changing the ways of relationships and contact between people, reaching the occupation sphere, replacing e realocating the work, before, done by human strengh and cognition, this growing force is called Artificial Intelligence, and it's constant technological advances generate urgency of it's study to achieve a better comprehension. This paper is destined to show the operation and classification of artificial intelligent systems, executing explanations of presente concepts in Artificial Intelligence. This analysis was made seeking to understand the artificial intelligent systems utilized by the Judiciary Branch, their classifications inside Artificial Intelligence, and if these are being

\footnotetext{
Pós-graduando na pontifícia Universidade Católica do Paraná (PUC-PR), Curitiba, Paraná, Brasil. Bacharel em direito, pela Universidade Estadual de Ponta Grossa -UEPG, Paraná, Brasil. Email: matheus.viezzer@hotmail.com
} 


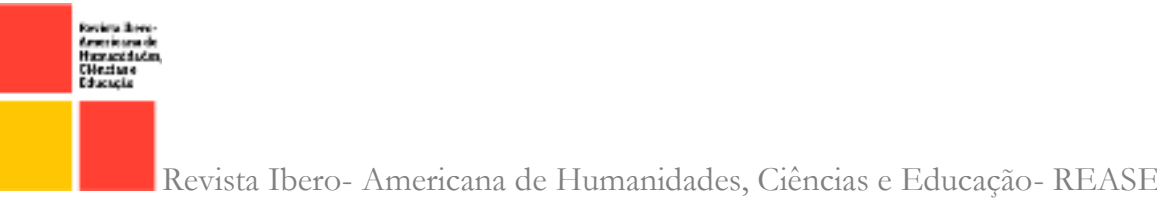

utilized in a way that comprehends all of it's abilities and features. This paper utilized it self of the review of specific literary material, as well as studies in sites of each project ou concept analyzed, performing bibliographic and descriptive research, reviewing the subjects with more cientific approval, and discussing the applications of those conceptions about the model utilized by the Judiciary Branch, aiming to verify if they use Artifical Intelligence or are only utilized to automation tasks.

Keywords: Technological advances. Artificial Intelligence. Comprehension. Classification. Judiciary Branch.

\section{INTRODUÇÃO}

Desde o surgimento do primeiro mecanismo computadorizado, desenvolvido por Alan Mathison Turing, e da criação do primeiro propriamente dito sistema computadorizado, também por Turing, estudiosos apresentam que estas máquinas compreenderão todas as funções cognitivas do cérebro humano, como descreve Nick Bostrom em sua obra Superintelligence - paths, dangers and strategies: "Machines matching humans in general intelligence $[\ldots]$ have been expected since the invention of computers in the 1940s. At that time the advent of such machines was often placed some twenty years into the future." 2

A ideia de alcançar um sistema inteligente artificialmente, o qual consiga se equiparar à inteligência humana, vem trazendo diversos avanços neste campo, onde cada dia surgem novas funções do cérebro humano reproduzidas por componentes artificialmente inteligentes.

Sendo estas tecnologias cada vez mais utilizadas no mercado de trabalho, substituindo atividades previamente realizadas por seres humanos por sistemas robotizados inteligentes, e este caminho também vêm sendo traçado dentro da esfera jurídica, onde, pelo mundo e aqui no Brasil, a implementação destes trazem celeridade e até mesmo maior assertividade em dados aspectos.

Restando por extremamente importante, compreendermos o funcionamento da Inteligência Artificial, afim de entendermos qual o futuro e quão próximo este está. Sendo

\footnotetext{
${ }^{2}$ Máquinas se igualando a inteligência humana [...] é algo esperado desde a invenção de computadores na década de 1940. Nessa época o advento de tais máquinas eram projetadas para vinte anos no futuro. (BOSTROM, Nick. Superintelligence Paths, Dangers, Strategies. Oxônia: OUP Oxford, 20r6. p. 4, tradução livre)
} 
realizada análise de três sistemas atualmente utilizados pelo Poder Judiciário brasileiro, sendo eles o projeto ELIS, o projeto POTI e o projeto VICTOR.

Esta análise busca por fim realizar a diferenciação da automação de serviços judiciários e a aplicação da Inteligência Artificial, verificando se os algoritmos utilizados nestes projetos se caracterizam como artificialmente inteligentes e se realizam todas as funções a eles possíveis de execução.

\section{INTELIGÊNCIA ARTIFICIAL}

Definir de forma única o significado de Inteligência Artificial não ocorre de forma fácil, em razão de que, os sistemas assim denominados, abrangem diversos outros, as quais podemos denominar de subcategorias. Assim, é necessário destacar que a doutrina especializada, até os dias atuais, não alcançou definição única abrangendo todo este instituto. Desta forma, afim de atingir melhor compreensão a respeito do que é a Inteligência Artificial, trazem-se conceituações diversas, as quais convergem na tentativa de explicar de forma adequada este tema.

De início, aponta-se definição exposta por Fabiano Hartmann Peixoto e Roberta Zumblick Martins da Silva, em sua obra Inteligência Artificial e Direito, os quais, para realizar tal conceituação, fazem uso das definições de Miles Brudage sobre o tema, dispondo:

Miles Brundage (2018) define IA como um corpo de pesquisa e engenharia com o objetivo de usar a tecnologia digital para criar sistemas aptos a desempenhar atividades para as quais se costuma exigir inteligência, ao ser realizada por um indivíduo. ${ }^{3}$

Para Miles Brudage, a Inteligência Artificial é um ramo de estudo onde convergem a computação e a engenharia, com o intuito de alcançar a criação de sistemas inteligentes para realizarem tarefas hoje fornecidas a um ser humano as quais exijam dele dado esforço mental. O preenchimento deste requisito qualifica o sistema em análise como inteligente.

A tal definição, acrescenta-se a definição apresentada por Jahanzaib Shabbir e Tarique Anwer, no artigo publicado denominado Artificial Intelligence and its Role in Near

\footnotetext{
${ }^{3}$ PEIXOTO, Fabiano Hartmann; ZUMBLICK, Martins da Silva. Inteligência Artificial e Direito. I. ed. Curitiba: Alteridade Editora, 2019. p. 21.
} 
Future, onde resta definida como Inteligência Artificial, os sistemas os quais apresentarem a habilidade de simular o pensamento de forma similar a inteligência de um ser humano, sendo possibilitado que estes resolvam problemas e tomem decisões, assim como possam aprender com seus próprios erros e acertos.

Artificial Intelligence is an efficient means that make computers and software control robotic thinking with expert systems that significantly illustrate the inteligente behavior, learning and effectievely advice user. In general, AI is basically known as the ability or potential of robotics to decide, solve problems and reason. ${ }^{4}$

De forma a unir as concepções previamente dispostas, expõe-se definição da empresa IBM (International Business Machine Corporation), desenvolvedora do principal sistema computadorizado em atividade, o supercomputador Watson, além de uma das principais empresas de software e sistemas inteligentes do mundo, dispõe:

Artificial intelligence refers to any human-like intelligence exhibited by a computer, robot, or other machine. It includes any application or tool that makes decisions and creates a corresponding output based on rules, input, and experience. ${ }^{5}$

Detém-se, desta forma, a denominação Inteligência Artificial deve ocorrer quando o sistema inteligente computadorizado apresentar semelhança ao comportamento da inteligência humana, a qual deve ser baseada em experiências, siga regras e comandos, e tome decisões, podendo estas últimas serem pré-determinadas pela ação humana ou não.

Perante tais conceitos, destaca-se o apontamento a respeito de um sistema inteligente auxiliado pelo ser humano, onde dadas vezes o ponto de partida, os resultados e respostas desejados são pré-determinados, serem classificados como Inteligência Artificial.

Esta diferenciação realizada, separa o instituto da Inteligência Artificial entre Fraca e Forte. Restando por necessária, assim, exposição contendo melhores diferenciações entre estas, o que se passa a fazer em sequência.

\footnotetext{
${ }^{4}$ Inteligência Artificial é um meio eficiente que faz com que computadores e softwares controlem o pensamento robótico com sistemas inteligentes que significativamente ilustram o comportamento inteligente, aprendendo e efetivamente auxiliando e orientando usuários. Em geral, IA é basicamente conhecida como a habilidade ou potencial da robótica em tomar decisões, resolver problemas e raciocinar. (SHABBER, Jahanzaib; ANWER, Tarique. Artificial Intelligence and its Role in Near Future. 2015, tradução livre).

${ }^{5}$ Inteligência Artificial se refere a qualquer inteligência similar a humana, exposta por um computador, robô ou qualquer outra máquina. Inclui-se qualquer aplicação ou ferramenta que toma decisões e cria uma resposta correspondente em regras, dados inseridos ou por sua experiência. (IBM. What is artificial intelligence?, tradução livre).
} 


\section{I. Inteligência artificial específica ou "fraca"}

Inteligência Artificial Fraca, também chamada de Inteligência Artificial Limitada, e Inteligência Limitada Aplicada, ou Inteligência Artificial Específica, é utilizada afim de realizar tarefas específicas, previamente definidas pelo homem. Porém, apesar do "final do caminho" ser determinado anteriormente, o trajeto percorrido por este sistema para a tomada de decisão adequada ou resolução de dado problema, é variável e independente.

Atualmente, estes sistemas são os mais utilizados no mundo, como exemplos destes, que utilizam a Inteligência Limitada temos: a Siri, aplicativo de assistência da Apple, Cortana, aplicativo também assistencial dos sistemas Windows, o Alexa, da Amazon e diversos outros, como detém-se de artigo presente em sítio eletrônico da empresa IBM, "Weak AI - also called Narrow AI or Artificial Narrow Intelligence (ANI )is AI trained and focused to perform specific tasks. Weak AI drives most of the AI that surrounds us today."

Verifica-se que estes sistemas já possuem em sua programação limitação para com as respostas que podem fornecer e os dados a serem utilizados, porém, o caminho percorrido pelo sistema para atingir as resoluções das problemáticas apresentadas é controlado pelo próprio,

Entende-se, assim que, a Inteligência Artificial definida como "Fraca", não consegue realizar conexões lógicas por sua própria vontade, de forma que, resta necessária a iniciativa humana para seu desenvolver. Por esse motivo, muitos especialistas não consideram esta mesma como Inteligência Artificial propriamente dita. Porém, para outros, o termo "Fraca" não deveria ser utilizado, mas sim os temos o termo "Limitada" ou "Específica" indicaria melhor as situações que estes sistemas são aplicados.

De forma mais incisiva, sistemas assim classificados são caracterizados pela necessidade da ação humana para realizar dada função, ou seja, as atividades efetuadas são limitadas pelo código arquitetado para ela, sem esta apresentar outro tipo de faculdade para realizar tarefas não escritas ou diversas das constantes em seu código base.

\footnotetext{
${ }^{6}$ Inteligência Artificial se refere a qualquer inteligência similar a humana, exposta por um computador, robô ou qualquer outra máquina. Inclui-se qualquer aplicação ou ferramenta que toma decisões e cria uma resposta correspondente em regras, dados inseridos ou por sua experiência. (IBM. What is artificial intelligence?, tradução livre).
} 
Devendo, por assim, ser afastada a concepção de fraqueza destes, pois apesar de limitadamente, são sistemas inteligentes. Resta por necessário, destacar que a maioria dos sistemas utilizados e presentes em nossa vida cotidiana se utilizam de inteligência classificada como "Limitada", como outros exemplos têm-se dispositivos como: celulares, computadores e televisores, além de utilização em empresas, afim de realizar funções repetitivas e sem necessidade de raciocínio lógico.

A nomenclatura concedida de "Fraca" ou "Limitada", se dá, também, em razão da existência da chamada Inteligência Artificial "Geral" ou "Forte". Sistemas inteligentes os quais apresentam lógica e racionalidade para a solução de problemas e tarefas sem a influência humana no regimento de códigos de resposta.

\subsection{Inteligência artificial geral ou "forte"}

Esta, diferentemente da "Limitada", apresenta as funções similares as de um cérebro humano em toda sua complexidade. Sendo assim, esta mesma é capaz de desenvolver-se, analisar questões por conta própria, usando somente um código base, aprendendo a assimilar este mesmo e estabelecendo comportamentos diferentes dos impostos pelo mesmo código, como deixa claro a definição exposta no sítio eletrônico oficial da IBM sobre o tema Strong AI (Inteligência Artificial Forte): "If researchers are able to develop Strong AI, the machine would require an intelligence equal to humans; it would have a self-aware consciousness that has the ability to solve problems, learn, and plan for the future." 7

Afim de melhor explicitar este conceito, citam-se estudos de $\mathrm{Zi}$ Wang, Leslie Kaelbling e Tomas Lozano-Perez. Estes trabalharam no desenvolvimento de um sistema de apoio domiciliar, o qual realizaria a leitura e compreensão de diversos casos diferentes, com peculiaridades difíceis de serem redigidas por código, devido à infinidade de situações as quais tal sistema estaria exposto. Como apresenta Fabiano Hartmann Peixoto, ao tratar deste tema em seu livro Inteligência Artificial e Direito: Convergência Ética e Estratégica.

Cabe ao sistema de inteligência entender como as coisas funcionam e alimentar um sistema preditivo para fazer escolhas eficazes e seguras. Então, ao menos sob

\footnotetext{
${ }^{7}$ Se pesquisadores forem capazes de desenvolver a Inteligência Artificial Forte, tal máquina exigiria uma inteligência igual a humana; a mesma teria uma consciência de si mesmo que seria capaz de resolver problemas, aprender e realizar planos para o futuro. (IBM. What is artificial intelligence?, tradução livre).
} 
uma lógica incipiente, é possível se associas IA a decisões complexas imprevistas com possibilidade de interferências fortes nos desígnios de pessoas. ${ }^{8}$

Frente a cognição presente em tais sistemas, parte majoritária da doutrina especializada, considera esta, como Inteligência Artificial. Adotando este tratamento, conclui-se que somente alguns sistemas denominados inteligentes artificialmente, assim podem ser definidos, para estes, o conceito de inteligência abordado anteriormente não deve ser considerado Inteligência Artificial, mas somente uma de suas aplicações.

Ainda, é exposto pela doutrina majoritária que dado sistema que supostamente contém Inteligência Artificial Forte, somente poderia ser assim classificado, nos casos em que a máquina ou sistema possa aprender sozinha, sem a necessidade de intervenções humanas para manter-se atualizada e execute diversas funções, aprendendo com base no ambiente em que se encontra. Como expõe Ondrej Kubovic com a contribuição de Peter Kosinar e Juraj Jánosik, no artigo Can Artificial Intelligence Power Future Malware?.

The idea of artificial intelligence (AI) has been around for more than 6o years. It represents the yet unachievable ideal of a generally intelligent and selfsustainable machine that can learn independently, based on inputs from the environment. ${ }^{9}$

Os mesmos autores, no mesmo artigo publicado, apresentam que nos dias atuais, somente tratamos de uma das subcategorias da Inteligência Artificial, a Machine Learning, porém, este método de aprendizado do sistema não pode ser confundido com o conceito principal, visto que, esta é apenas uma subcategoria de Inteligência Artificial "Forte": "Yet, today a "AI" often refers only to a subcategory of this technology - namely machine learning $(M L)$." iо

Afim de compreender a possibilidade de existência de dado sistema possuidor de Inteligência Artificial "Forte", John Searle, apresentou a teoria do Quarto Chinês. Esta teoria dispõe sobre a seguinte hipótese: Em uma sala se encontra uma pessoa que não fala

\footnotetext{
${ }^{8}$ PEIXOTO, Fabiano Hartmann. Inteligência Artificial e Direito: Convergência Ética e Estratégica. vol. o5. ed. or. Curitiba: Alteridade Editora, 2020. p. 28

${ }^{9} \mathrm{~A}$ ideia de Inteligência Artificial (IA) está presente por mais de 60 anos. Ela representa o ainda inalcançável ideal de uma máquina inteligente em todas as frentes e autossustentável que poderia aprender independentemente, baseada apenas em informações do ambiente em que esta se encontra. (KUBOVIC, Ondrej; KOSINAR, Peter; JÁNOSIK, Juraj. Can Artificial Intelligence Power Future Malware?, tradução livre)

ro Porém, "IA" hoje comumente se refere apenas a uma das subcategorias desta tecnologia - nomeada aprendizado de máquina (machine leaning (ML)). (Ibid., tradução livre)
} 
chinês, neste cômodo que ela está, se encontra um livro com as regras e frases em chinês. Uma pessoa fluente em chinês, e fora da sala, começa a passar diversas anotações e perguntas em chinês para a primeira pessoa. Com a ajuda do livro, a pessoa dentro da sala responde tais perguntas de forma correta, porém, mesmo acertando as respostas, não significa que ela aprendeu chinês.

Com base nesta teoria, Searle apresenta para a existência da Inteligência Artificial "Forte", esta mesma deve possuir mente própria, e ter certa consciência, para que consiga elaborar entendimentos e respostas sobre dados aspectos, porém, para este Autor, tal nível nunca será alcançado, pois o sistema não possui a capacidade de perceber o lado semântico.

Computation is defined purely formally or syntactically, whereas minds have actual mental or semantic contents, and we cannot get from syntactical to the semantic just by having the syntactical operations and nothing else...A system, me, for example, would not acquire an understanding of Chinese just by going through the steps of a computer program that simulated the behavior of a Chinese speaker ${ }^{\text {II }}$.

David Chalmers e Habs Moravec adentram a discussão levantada por Searle, e apresentam, de forma contrária a teoria apresentada anteriormente, a possibilidade da criação de um sistema inteligente que consiga formular decisões por conta própria, aprendendo sozinho, fugindo do estabelecido por seu código base, trazendo como exemplo a dominação das leis da física pela sociedade humana, para fazer um avião voar, a sociedade humana conseguirá atuar de forma a dominar este tipo de sistema.

The existence of several examples of intelligence designed under these constraints should give us great confidence that we can achieve the same in short order. The situation is analogous to the history of heavier than air flight, where birds, bats and insects clearly demonstrated the possibility before our culture mastered it. ${ }^{\text {I2 }}$

\footnotetext{
II Computação é definida de forma puramente formal ou sinteticamente, porém as mentes possuem conteúdos mentais ou semânticos, e nós não conseguimos fazer com que o sintético alcance o semântico, fazendo com que o sistema realize apenas operações sintéticas e nada mais... Um sistema, eu, por exemplo, não conseguiria adquirir conhecimento de chinês apenas seguindo os passos de um programa de computador que simula o comportamento de uma pessoa que fale chinês. (SEARLE, John. Minds, Brains and Science. ed. 13. Harvard University Press, 2003. p. 17, tradução livre)

${ }_{12}$ A existência de diversos exemplos de inteligência baseada nestes conceitos, deveria nos fornecer grande confiança de que nós podemos alcançar o mesmo em um futuro próximo. A situação é análoga a história do primeiro voo, onde pássaros, morcegos e insetos já haviam demonstrado a possibilidade que objetos mais pesados que o ar pudessem voar, antes do ser humano dominar tal arte. (MORAVEC, Hans P. The role of raw power in intelligence, 1976 , tradução livre)
} 
Assim sendo, como o ser humano conseguiu dominar as leis da física, fazendo com que a engenharia realizasse feitos antes inimagináveis, a criação de um sistema inteligente que supere os dados transcritos em sua base e que limite suas atividades, é extremamente plausível. Tais autores não sabiam quanto tempo seria necessário para serem alcançados tais sistemas, porém, estas afirmações aumentaram a crença de que dispositivos providos destas características teriam origem.

\subsection{Machine learning e deep learning}

Machine Learning, de forma livremente traduzida, representa o aprendizado de máquina, forma na qual o algoritmo presente em dispositivos inteligentes aprende com o meio em que se encontra.

Para Isabela Ferrari e Daniel Becker, na obra - Obra Inteligência Artificial E Direito Processual - Os impactos da virada tecnológica no direito processual ${ }^{24}$, se utilizando da definição exposta por K. Murphy, este componente da Inteligência Artificial pode ser definido da seguinte forma:

Podemos definir a técnica de machine learning, então, como a prática de usar algoritmos para coletar e interpretar dados, fazendo predições sobre fenômenos, de forma que as máquinas desenvolvam os próprios modelos e façam predições automáticas, independentemente de nova programação. ${ }^{13}$

Frente a tal conceito, entende-se que, realizando técnicas destinadas a aprendizado, o algoritmo, coleta e interpreta dados, porém, não somente para realizar tomada de decisão, mas o faz sem necessidade de intervenção humana para que seja alcançada nova programação ou definição da solução do problema. De forma que, quando destinada a solução de um problema, o sistema desenvolva cognição para a resolução do mesmo, estabelecendo aprendizado, não somente sobre a resposta de tal problema, mas também sobre o todo que cerca este.

Com isto, traz-se definição trazida por Nick Bostrom, onde o mesmo desenvolveu a teoria da inteligência como uma semente. Nesta, o algoritmo seria capaz de se alterar e se desenvolver, de acordo com as formas de aprendizado (tentativa e erro) e coleta de

\footnotetext{
${ }^{13}$ FERRARI, Isabela; BECKER, Daniel. Direito à explicação e decisões automatizadas: reflexões sobre o princípio do contraditório. Inteligência Artificial e o Direito Processual. 2. ed. Editora JusPodivim, 2021.
} 
informações e conhecimento pré-existentes, de forma que, este sistema seria capaz de entender suas próprias habilidades.

A variation on Turing's conception of a child machine is the idea of a "seed AI." Whereas a child machine, as Turing seems to have envisaged it, would have a relatively fixed architecture that simply develops its inherent potentialities by accumulating content, a seed AI would be a more sophisticated artificial intelligence capable of improving its own architecture. In the early stages of a seed AI, such improvements might occur mainly through trial and error, information acquisition, or assistance from the programmers. At its later stages, however, a seed AI should be able to understand its own workings sufficiently to engineer new algorithms and computational structures to bootstrap its cognitive performance. ${ }^{14}$

Restando por necessária a distinção do aprendizado de máquina e o aprendizado profundo, denominado Deep Learning. Usa-se a conceituação elaborada pelos autores Ian Goodfelow, Yosgua Bengio e Aaron Courville, em sua obra chamada Deep Learning.

The true challenge to artificial intelligence proved to be solving the tasks that are easy for people to perform but hard for people to describe formally problems that we solve intuitively, that feel automatic, like recognizing spoken words or faces in images. [...]. This solution is to allow computers to learn from experience and understand the world in terms of a hierarchy of concepts, with each concept defined through its relation to simpler concepts. By gathering knowledge from experience, this approach avoids the need for human operators to formally specify all the knowledge that the computer needs. The hierarchy of concepts enables the computer to learn complicated concepts by building them out of simpler ones. If we draw a graph showing how these concepts are built on top of each other, the graph is deep, with many layers. For this reason, we call this approach to AI deep learning. ${ }^{\text {is }}$

${ }^{14}$ Uma variação do conceito de Turing de uma inteligência de uma criança, é a ideia de uma IA semente. Onde uma inteligência como de uma criança, como Turing parece ter previsto, teria uma arquitetura relativamente fixa que somente desenvolve sua potencialidade inerente acumulando conhecimento, a IA semente seria uma Inteligência Artificial mais sofisticada, capaz de modificar sua própria arquitetura. Nos primeiros estágios da IA semente, tais melhorias podem ocorrer, principalmente, por meio da tentativa e erro, aquisição de informação ou assistência de programadores. Em seus estágios posteriores, entretanto, a IA semente seria capaz de entender seu próprio funcionamento suficientemente para desenvolver novos algoritmos e estruturas computadorizadas para dar início a sua performance cognitiva. (BOSTROM, Nick. Superintelligence Paths, Dangers, Strategies. Oxônia: OUP Oxford, 20ı6., p. 34, tradução livre)

${ }^{15} \mathrm{O}$ verdadeiro desafio para a Inteligência Artificial se provou ser a resolução de tarefas que são fáceis para os seres humanos executar, mas difíceis de serem descritos de uma maneira formal, problemas que nós resolvemos intuitivamente, que são automáticos, como reconhecimento de fala ou de rostos em uma imagem [...]. A solução é permitir que computadores aprendam pela experiência e entendam o mundo em termos de hierarquia de conceitos, com cada conceito definido pela sua relação com conceitos simples. Acumulando conhecimento pela experiência, essa abordagem evita a necessidade de operadores humanos de especificar todo o conhecimento que o computador precisa. A hierarquia de conceitos possibilita ao computador aprender conceitos complicados, ao construí-los a partir de conceitos mais simples. Se desenhássemos um gráfico apresentando como estes conceitos são formados, um sobre o outro, ele seria profundo, com muitas camadas. Por esta razão, nós chamamos esta abordagem da Inteligência Artificial de deep learning 
De acordo com estes ensinamentos, entendemos que a Deep Learning é derivada da Machine Learning, visto que, quando da utilização desta, não é necessária a intervenção humana para possibilitar o aprendizado do sistema desenvolvido, sendo que, o aprendizado se daria pelo sistema de tentativa e erro, por conta própria do algoritmo inteligente.

Detém-se da conceituação apresentada por Ian Goodfelow, Yosgua Bengio e Aaron Courville, que a aplicação deste instituto se dá com a utilização de algoritmos destinados a realizar funções consideradas fáceis aos seres humanos, porém extremamente complexas de serem transcritas para o aprendizado e execução dos sistemas inteligentes. Tais funções ocorrem quando os algoritmos desconstroem as camadas de dado problema (input), produzindo diversos conceitos simples, e a partir destes, realiza agrupamentos, desenvolvendo um conceito complexo e alcançando o resultado desejado (output). Como pode ser observado na imagem apresentada por Goodfelow, Bengio e Courville, nesta mesma obra destacada.

Figura demonstrativa do funcionamento do sistema Deep Learning. ${ }^{16}$

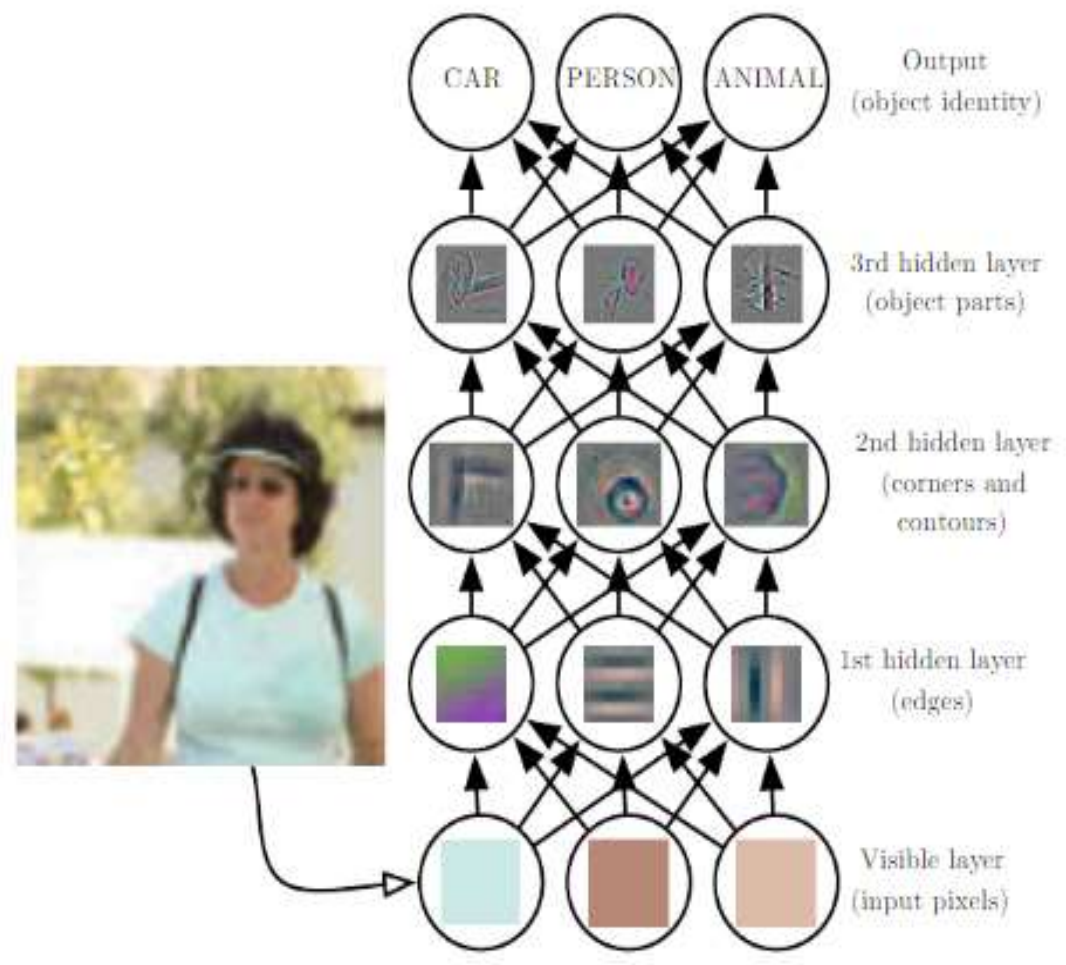

(aprendizagem profunda). (GOODFELlOW, Ian; BENGIO, Yoshua; COURVILlE, Aaron. Deep Learning. 20I6, p. oI, tradução livre)

${ }^{16}$ Fonte: Ian Goodfelow, Yosgua Bengio e Aaron Courville, em sua obra chamada Deep Learning - pg. 6. 
Note-se que na imagem são dispostas três camadas escondidas (hidden layers), porém, deve-se destacar que esta imagem é apenas ilustrativa, afim de representar o caminho dos dados captados pelo sistema, em razão de que, a rede neural artificial possui incontáveis camadas, não sendo possível precisar exatamente quantas são utilizadas para cada raciocínio, sabendo-se somente que, quanto mais pesada a informação fornecida e desejada, mais interconexões ocorrem. Como apresentam os ensinamentos de José Luis Bolzan de Morais e Flaviane de Magalhães Barros, eu sua obra Compartilhamento de Dados e Devido Processo:

As redes neurais artificias são reconhecidas pelos próprios experts como "caixas pretas", em especial, pela não possibilidade de conhecer os caminhos que os neurônios artificias da camada intermediária ao longo do treinamento podem passar. ${ }^{17}$

Podemos, assim, compreender a Deep Learning como um sistema integrante da Machine Learning, pois como apresentado, se destina ao aprendizado do algoritmo de funções relativamente fáceis para a cognição humana, porém complexas para serem transformadas em um código para a interpretação da máquina, de forma que, o algoritmo divide esta atividade complexa em várias outras mais simples, depois juntando-as e gerando uma resposta. De forma a alcançar, não somente a resposta desejada (output) ou o aprendizado ensejado por seus desenvolvedores, mas sim, o aprendizado de todo o ambiente em qual se encontra, de forma autônoma.

\section{SISTEMAS UTILIZADOS PELO PODER JUDICIÁRIO BRASILEIRO}

Perante todas as definições trazidas anteriormente dispostas, passa-se as expor alguns dos sistemas inteligentes utilizados pelo Poder Judiciário brasileiro.

\subsection{Sistema Elis}

Este projeto foi o primeiro produto da Comissão para a Aplicação de Soluções em Inteligência Artificial (CIA) do Tribunal de Justiça do Estado de Pernambuco. Tal

\footnotetext{
${ }^{17}$ BARROS, Flaviane de Magalhães; MORAIS, José Bolzan. Compartilhamento de Dados e Devido Processo: Como o uso da inteligência artificial pode implicar em uma verdadeira aleteica. ed. or. Salvador: Editora JusPodivim. p. 276.
} 
sistema, desenvolvido pela SETIC (Secretaria de Tecnologia da Informação e Comunicação), é destinado a análise e a triagem dos processos executivos fiscais no estado de Pernambuco.

A tal programa, fora ensinado a realizar fiscalização e triagem dos processos presentes no meio eletrônico da Prefeitura de Recife, Pernambuco, tendo como input ações selecionadas pela Vara de Executivos Fiscais da Capital. Mediante esta base de dados fornecida, o algoritmo aprendeu a realizar a classificação destes processos nos que continham divergências cadastrais, competências diversas e eventuais prescrições. Em suas primeiras simulações, de acordo com o Tribunal de Justiça do Estado de Pernambuco, por meio do diretor de sistemas do Tribunal, Raphael José D'Castro, o sistema realizou análise de 5.247 processos.

Na simulação realizada pela Setic em um ambiente de homologação, o sistema
"ELIS" avaliou 5.247 processos e conseguiu classificar com precisão a
competência das ações, divergências cadastrais, erros no cadastro de dívida ativa e
casos de prescrição. "Desse total de ações judiciais distribuídas eletronicamente,
4.447 ( $84 \%$ ) estavam aptas a continuar tramitando; 640 (12\%) foram ajuizadas,
mas estavam prescritas; i6o (3\%) continham algum erro na certidão de dívida
ativa (CDA); i6 (o,3\%) foram incorretamente distribuídas porque eram de
competência estadual e 14 (o,3\%) continham dados divergentes. Em três dias,
"ELIS" foi capaz de fazer a triagem de mais de 5 mil processos. ${ }^{8}$

Diante dos dados fornecidos, destacam-se as classificações resultantes da análise, sendo estas, ações de execução aptas para a tramitação, ações ajuizadas, porém prescritas, ações contendo erro na certidão de dívida ativa, ações distribuídas de forma incorreta, em razão de erro quanto a competência e ações contendo dados divergentes. Perante esta classificação realizada, devemos destacar a velocidade com que esta análise se deu, apenas três dias foram necessários para a análise de mais de 5 mil processos.

Engrandecendo mais a resposta obtida, o juiz de Direito José Faustino Macêdo de Souza Ferreira, integrante da CIA TJPE, apresentou que o tempo necessário para a ser realizada a triagem e movimentação processual de $80 \mathrm{mil}$ processos seria de aproximadamente 18 meses quando por via humana, em perspectiva, para a análise desta mesma quantia de processos, o sistema ELIS seria capaz de realizar tal feito em aproximadamente is dias.

\footnotetext{
${ }^{18}$ BRITO, Bruno; TJPE, Ascom. TJPE usará inteligência artificial para agilizar processos de execução fiscal no Recife. Acessado em 28 de janeiro de 2022.
} 
Antes do desenvolvimento de 'ELIS', era necessário designar servidores para fazer a análise e a triagem individual da certidão de dívida ativa e da petição inicial. Em sequência, essa equipe pôde minutar e despachar cada um dos processos. Esse procedimento, com o trabalho exclusivamente de humanos, consome aproximadamente 18 meses para a triagem e movimentação processual de 80 mil feitos. 'ELIS' consegue realizar, com maior acurácia, a triagem da mesma quantidade de ações judiciais em is dias, ou em até menos, segundo nossa perspectiva. ${ }^{19}$

Ademais, fora apresentado, em conjunto a tais dados, a capacidade do ELIS em desenvolver e inserir minutas, assim como, se o magistrado assim deseja, assinar despachos proferidos pelo mesmo. Esta função seria adicionada ao algoritmo em uma segunda etapa ${ }^{27}$.

Tais informações, destacam a relevante diferença oriunda da implementação deste sistema, auxiliando o Poder Judiciário a alcançar celeridade processual e de forma subsequente, ocasionando melhor aproveitamento da parte humana do sistema jurídico, em razão de redistribuição de funções dos prestadores de serviços.

\subsection{Sistema Poti}

Desenvolvido pelo Tribunal de Justiça do Estado do Rio Grande do Norte (TJRN) em parceria com a Universidade Federal do Rio Grande do Norte (UFRN), sistema Poti se destina a execução de tarefas de bloqueio e desbloqueio de contas, assim como, à tarefa de emissão de certidões relativas ao BACENJUD (Sistema de Comunicação Eletrônica entre o Poder Judiciário e instituições financeiras e demais entidades autorizadas pelo Banco Central).

Este sistema realiza a junção do Poder Judiciário, Banco Central e as instituições bancárias, buscando a celeridade para a solicitação de informações e envios de ordens judiciais ao Sistema Financeiro Nacional, tornando a distância existente anteriormente entre estas instituições, menor e direta. Como extrai-se dos apontamentos feitos por Têmis Limberger e Demétrio Beck da Silva Giannakos, em artigo denominado O Princípio Constitucional da Eficiência e a Transparência, analisados sob a Ótica do Custo da Justiça: como aprimoramento da responsabilidade da entrega da prestação jurisdicional.

\footnotetext{
${ }^{19}$ BRITO, Bruno; TJPE, Ascom. TJPE usará inteligência artificial para agilizar processos de execução fiscal no Recife. Acessado em 28 de janeiro de 2022.
} 
Poti, em que executa tarefas de bloqueio, desbloqueio de valores em contas e emissão de certidões relacionadas ao Bacenjud (é um sistema que interliga a Justiça, o Banco Central e as instituições bancárias, para agilizar a solicitação de informações e o envio de ordens judiciais ao Sistema Financeiro Nacional, via internet). Tais tarefas, quando realizadas por servidores do Poder Judiciário, levavam semanas. Agora, são realizadas em segundos. ${ }^{20}$

Além das funções deste sistema, os autores em destaque, apontam que tarefas incumbidas ao sistema, são realizadas em segundos, antes de sua aplicação, os servidores destinados a prática destas mesmas funções, levavam semanas para as concluírem. Resultando, assim como a implementação do sistema ELIS, na melhor utilização do esforço humano presente no Poder Judiciário.

\subsection{Sistema Victor}

O desenvolvimento deste projeto se deu em razão de parceria entre o Supremo Tribunal Federal e a Universidade de Brasília (UnB), sendo que três cursos de graduação se ocuparam da realização deste projeto, curso de Direito, Engenharia de Software e Ciência da Computação.

Trata-se de projeto que envolve a parceria entre três cursos da UnB: Direito, Engenharia de Software e Ciência da Computação. Como assinalado, o projeto tem por objeto a aplicação de métodos de aprendizado computacional de máquina com o objetivo de usar seus potenciais no reconhecimento de padrões nos processos jurídicos relativos a julgamentos de repercussão geral do STF. ${ }^{21}$

Para compreendermos de forma adequada o funcionamento deste sistema, devemos compreender quais as funções executadas pelo mesmo, descritas no sítio eletrônico do Supremo Tribunal Federal.

O objetivo inicial é aumentar a velocidade de tramitação dos processos por meio da utilização da tecnologia para auxiliar o trabalho do Supremo Tribunal. A máquina não decide, não julga, isso é atividade humana. Está sendo treinado para atuar em camadas de organização dos processos para aumentar a eficiência e velocidade de avaliação judicial. ${ }^{22}$

\footnotetext{
${ }^{20}$ LIMBERGER, Têmis; GIANNAKOS, Demétrio Beck da Silva. O Princípio Constitucional da Eficiência e a Transparência, Analisados sob a Ótica do Custo da Justiça: como aprimoramento da responsabilidade da entrega da prestação jurisdicional. Revista Eletrônica CNJ, v. 3, n. oı, 2019, p. o7.

${ }^{21}$ MAIA FILHO, Mamede Said; JUNQUILHO, Tainá Aguiar. Projeto VICTOR: Perspectivas de aplicação da inteligência artificial ao direito. Revista de Direitos e Garantias Fundamentais. volume 19. n. 3, p. 225. Vitória, 2018.

${ }^{22}$ Supremo Tribunal Federal. Inteligência artificial vai agilizar a tramitação de processos no STF, 2018.
} 
Assim, o sistema será aplicado para exercer as funções de análise e classificação de processos, buscando a celeridade nas atividades do STF como um todo, afastando a hipótese de aplicação deste sistema para a tomada de decisões em julgamentos, sendo resguardada esta mesma para o ser humano.

A respeito de seu funcionamento, este mesmo analisa base de dados (Big Data), onde estão disponíveis os modelos de recursos extraordinários e agravos de recursos extraordinários, escolhidos pela equipe a qual o arquitetou, para o aprendizado.

Após ser definida esta base de dados, o algoritmo é treinado afim de que este aprenda como realizar as distinções processuais a qual é destinado, por meio da análise de exemplos considerados de repercussão geral e exemplos que não se qualificam desta forma. Como ditam os autores Mamede Said Maia Filho e Tainá Aguiar Junquilho, na obra Projeto Victor: Perspectivas de Aplicação da Inteligência Artificial ao Direito.

Após o estudo dos dados das repercussões gerais para estruturação e preparação para treinamento dos modelos de aprendizado de máquina supervisionados e não supervisionados, o projeto desenvolverá pesquisa dos possíveis algoritmos e estratégias de treinamento mais eficientes para o contexto estudado, englobando redes neurais artificiais profundas, para, em seguida, realizar a prototipação e treinamento dos algoritmos escolhidos, incluindo a sua avaliação. ${ }^{23}$

Quando da fase de desenvolvimento do VICTOR, o algoritmo fora treinado sendo utilizados cerca de 14.000 (quatorze mil) processos. Onde este realizou a separação e a classificação das peças consideradas de maior importância, como acórdãos, recursos extraordinários, agravos de recursos extraordinários, despacho de admissibilidade e sentença, e identificando os temas onde a repercussão geral possuía maior incidência.

Os resultados demonstrados apresentam eficácia e assertividade na separação e classificação dos temas considerados de repercussão geral, mostrando elevada porcentagem de assertividade, como descreveram Mamede Said Maia Filho e Tainá Aguiar Junquilho.

Trabalhando com cerca de 14.000 processos, a equipe do projeto centrou-se, inicialmente, na tarefa de separação e classificação de peças - aquelas mais importantes, segundo a equipe do STF, para a identificação dos temas de repercussão geral de maior incidência: acórdão, recurso extraordinário, agravo em recurso extraordinário, despacho de admissibilidade e sentença. [...] Mediante a utilização de diferentes modelos de aprendizado de máquina, foram alcançados

\footnotetext{
${ }^{23}$ MAIA FILHO, Mamede Said; JUNQUILHO, Tainá Aguiar. Projeto VICTOR: Perspectivas de aplicação da inteligência artificial ao direito. Revista de Direitos e Garantias Fundamentais. volume 19. n. 3, p. 227. Vitória, 2018.
} 
elevados níveis de assertividade na separação de peças, o que faz com que a fase seguinte do projeto - a identificação e classificação dos temas de repercussão geral - apresente perspectivas promissoras de bons resultados, constatáveis quando se for relacionar um processo inteiro a um ou mais temas de repercussão geral. ${ }^{24}$

A base de dados utilizada pelo projeto, atualmente, é constituída por aproximadamente 952.000 (novecentos e cinquenta e dois mil) documentos, os quais são provenientes de aproximadamente 45.000 (quarenta e cinco mil) processos.

Posteriormente, o sistema realiza a filtragem dos documentos com certas falhas, como erros de digitação e imagens não legíveis. Após isto, as frases são selecionadas e divididas em diversas partes menores, separando quais destas são mais relevantes para o texto. A seguir, identifica palavras parecidas ou que compartilham o mesmo radical, e realiza a separação em arquivos, cada arquivo é etiquetado, sendo classificado ou não a uma das peças importantes para o sistema. Por fim, é atribuído, o rótulo de repercussão geral do processo.

Os arquivos são então submetidos a um fluxo de tratamento de documentos que: I - Filtra elementos considerados espúrios, como erros de digitalização e imagens; 2 - Divide frases em partes menores e cria símbolos para as partes mais relevantes do texto; 3 - Reduz palavras muito parecidas ou que possuem mesmo radical a símbolos comuns; 4 - Dá uma etiqueta a cada arquivo, classificando-o em uma das peças relevantes ao projeto; 5 - Atribui um rótulo com a repercussão geral do processo $^{25}$.

Parte-se para a análise dos resultados obtidos pela fase de testes do sistema e projetar os impactos futuros, trazidos pela implementação deste sistema. No ano de 2018, a ministra Carmen Lúcia, a época, presidente do Supremo Tribunal Federal, apresentou que o mesmo fora testado em 27 temas de repercussão geral, totalizando 60\% (sessenta por cento) de todos os temas atualmente encontrados no STF. O sistema obteve precisão de $84 \%$ (oitenta e quatro por cento) ao exercer suas funcionalidades, ainda, fora destacado que, sendo utilizando o aprendizado de máquina, o mesmo alcançaria 95\% (noventa e cinco por cento), no espaço de um mês.

Segundo informou a presidente, os testes com os processos envolvendo repercussão geral foram iniciados com 27 temas mais recorrentes no Tribunal,

\footnotetext{
${ }^{24}$ MAIA FILHO, Mamede Said; JUNQUILHO, Tainá Aguiar. Projeto VICTOR: Perspectivas de aplicação da inteligência artificial ao direito. Revista de Direitos e Garantias Fundamentais. volume 19. n. 3, p. 228. Vitória, 2018.

${ }^{25}$ INAZAWA, Pedro, et. al. Projeto VICTOR como o uso do aprendizado de máquina pode auxiliar a mais alta corte brasileira a aumentar a eficiência e a velocidade de avalição judicial dos processos julgados, 2019.
} 
representando 60\% do total de temas regularmente identificados. Ela informou que atualmente o nível de precisão na triagem é de $84 \%$, mas que atingirá $95 \%$ no próximo mês. ${ }^{26}$

A análise a todos os resultados obtidos, detém-se que a aplicação deste sistema resulte em redução temporal de aproximadamente 02 (dois) anos, em comparação ao tempo decorrente do trabalho humano.

Os pesquisadores e o Tribunal esperam que, em breve, todos os tribunais do Brasil poderão fazer uso do VICTOR para pré-processar os recursos extraordinários logo após sua interposição (esses recursos são interpostos contra acórdãos de tribunais), o que visa antecipar o juízo de admissibilidade quanto à vinculação a temas com repercussão geral, o primeiro obstáculo para que um recurso chegue ao STF. Com isso, poderá impactar na redução dessa fase em 2 ou mais anos. ${ }^{27}$

Em apresentação no seminário "Novas Tendências do Direito Comum Inteligência Artificial, Análise Econômica do Direito e Processo Civil”, no ano de 2019, o ex-presidente do Supremo Tribunal Federal, ministro Dias Toffoli, realizou declaração sobre a quantia de tempo necessária antes da implementação do sistema em comparação quando da sua utilização.

O programa VICTOR, que está em fase de estágio supervisionado, promete trazer maior eficiência na análise de processos, com economia de tempo e recursos humanos. [...] As tarefas que os servidores do tribunal levam, em média 44 minutos, o VICTOR fará em menos de 5 segundos. ${ }^{28}$

Verifica-se, assim, perante todo o apresentado, a importância da implementação deste sistema, ocupando posição de extrema valia para a condução dos processos de repercussão geral no Supremo Tribunal Federal.

O estudo dos dados apresentados a respeito deste sistema, conduzem ao entendimento da redução da quantia de tempo necessária para realizar determinados atos, ocasionando celeridade processual como todo, em razão da velocidade de execução de suas funções e devido a destinação do esforço dos servidores para atividades de maior exigência cognitiva. Sob este olhar, podemos definir que tais alterações no Poder Judiciário produziriam aprimoramento deste mesmo.

\footnotetext{
${ }^{26}$ STF. Presidente do Supremo apresenta ferramentas de inteligência artificial em Londres. 2018.

${ }^{27}$ STF. Ministra Cármen Lúcia anuncia início de funcionamento do Projeto Victor, de inteligência artificial, 2019.

${ }^{28}$ Id., Inteligência artificial vai agilizar a tramitação de processos no STF, 2019.
} 


\section{CONSIDERAÇÕES FINAIS}

Sob o olhar dos conceitos dispostos neste, fora realizada a análise dos sistemas utilizados pelo Poder Judiciário brasileiro, aqui elencados e explicitados, na forma de identificar se estes sistemas fazem uso de algoritmos inteligentes e classificá-los, como "Fortes" ou "Fracos", além de analisar se estes mesmos atuam como agentes de automação de função ou de forma a exercer sua inteligência.

Assim sendo, a doutrina majoritária identifica como comportamento de sistema necessário para classificação como sistema Inteligente Artificialmente "Forte", a presença de comportamentos similares ao do cérebro humano, somados à assimilação do ambiente em que se encontra e comportamentos diferentes dos estabelecidos em seu código base.

Com isto, pode-se estabelecer que os sistemas utilizados podem ser classificados como inteligentes, porém, este mesmo entendimento, leva a compreensão de que os sistemas atuantes no Poder Judiciário, aqui analisados, não podem ser classificados como "Fortes”, pois estes mesmos não apresentam as características necessárias para assim serem classificados.

Em segunda etapa, a análise para identificar se estes dispositivos inteligentes são utilizados de forma a compreender todas as suas capacidades ou se estes são utilizados de forma a automatizar processos previamente realizados pela cognição humana. Tal distinção, é possível em razão da classificação dos sistemas analisados em Inteligência Artificial "Limitada" ou "Fraca", visto que dispositivos utilizadores desta realizam tarefas limitadas de substituição de funções.

Conclui-se, assim, que os programas aqui analisados são providos de Inteligência Artificial "Fraca" ou "Limitada", e são utilizadas de forma a automatizar funções especificamente descritas, apresentando utilização do aprendizado de máquina (machine learning), porém, não sendo providos de conhecimento profundo (deep learning), não compreendendo, assim, funções diferentes das presentes em seu código principal, sem entender o ambiente em que se situa, somente realizando uma função específica. 


\section{REFERÊNCIAS}

BARROS, Flaviane de Magalhães; MORAIS, José Bolzan. Compartilhamento de Dados e Devido Processo: Como o uso da inteligência artificial pode implicar em uma verdadeira aleteica. ed. or. Salvador: Editora JusPodivim. p. 276;

BRITO, Bruno; TJPE, Ascom. TJPE usará inteligência artificial para agilizar processos de execução fiscal no Recife. $\langle$ http://www.tjpe.jus.br/inicio?p_p_id=IoI\&p_p_lifecycle=o\&p_p_state=maximized\&p_p $\rangle$ Acessado em 28 de janeiro de 2022;

BOSTROM, Nick. Superintelligence Paths, Dangers, Strategies. Oxônia: OUP Oxford, 2016. p. 4;

FERRARI, Isabela; BECKER, Daniel. Direito à explicação e decisões automatizadas: reflexões sobre o princípio do contraditório. Inteligência Artificial e o Direito Processual. 2. ed. Editora JusPodivim, 202I;

IBM. What is artificial intelligence?. Disponível em: <https://www.ibm.com/cloud/learn/what-is-artificial-

intelligence?mhsrc=ibmsearch_a\&mhq=narrow $>$ Acessado em 28 de janeiro de 2022;

INAZAWA, Pedro, et. al. Projeto VICTOR como o uso do aprendizado de máquina pode auxiliar a mais alta corte brasileira a aumentar a eficiência e a velocidade de avalição judicial dos processos julgados, 2019. Disponível em: 〈https://cic.unb.br/ teodecampos/ViP/inazawa_etal_compBrasil2or9.pdf> Acessado em 28 de janeiro de 2022;

GOODFELlOW, Ian; BENGIO, Yoshua; COURVILlE, Aaron. Deep Learning. 20i6, p. or;

KUBOVIC, Ondrej; KOSINAR, Peter; JÁNOSIK, Juraj. Can Artificial Intelligence Power Future Malware?. ESET;

LIMBERGER, Têmis; GIANNAKOS, Demétrio Beck da Silva. O Princípio Constitucional da Eficiência e a Transparência, Analisados sob a Ótica do Custo da Justiça: como aprimoramento da responsabilidade da entrega da prestação jurisdicional. Revista Eletrônica CNJ, v. 3, n. or, 2019, p. o7;

MAIA FILHO, Mamede Said; JUNQUILHO, Tainá Aguiar. Projeto VICTOR: Perspectivas de aplicação da inteligência artificial ao direito. Revista de Direitos e Garantias Fundamentais. volume 19. n. 3. Vitória, 2018;

MORAVEC, Hans $\mathrm{P}$. The role of raw power in intelligence, 1976 ;

PEIXOTO, Fabiano Hartmann; ZUMBLICK, Martins da Silva. Inteligência Artificial e Direito. I. ed. Curitiba: Alteridade Editora, 2019. p. 2I; 
PEIXOTO, Fabiano Hartmann. Inteligência Artificial e Direito: Convergência Ética e Estratégica. vol. 05. ed. or. Curitiba: Alteridade Editora, 2020. p. 28;

SEARLE, John. Minds, Brains and Science. ed. 13. Harvard University Press, 2003. p. 17;

SHABBER, Jahanzaib; ANWER, Tarique. Artificial Intelligence and its Role in Near Future. 2015;

Supremo Tribunal Federal. Inteligência artificial vai agilizar a tramitação de processos no STF, 2018. Disponível em: 〈https://portal.stf.jus.br/noticias/verNoticiaDetalhe.asp?idConteudo=380038\&ori=I〉 Acessado em 28 de janeiro de 2022;

Supremo Tribunal Federal. Ministra Cármen Lúcia anuncia início de funcionamento do Projeto Victor, de inteligência artificial. Disponível em: 〈https://portal.stf.jus.br/noticias/verNoticiaDetalhe.asp?idConteudo=388443\&ori=I > Acessado em 28 de janeiro de 2022;

Supremo Tribunal Federal. Inteligência artificial: Trabalho judicial de 40 minutos pode ser feito em 5 segundos. Disponível em: 〈https://portal.stf.jus.br/noticias/verNoticiaDetalhe.asp?idConteudo=393522\&ori=I > Acessado em 28 de janeiro de 2022; 